\title{
Future of epilepsy treatment: integration of devices
}

Amanda F Van Straten ${ }^{*, 1} \&$ Barbara C Jobst ${ }^{1}$

\begin{abstract}
The use of devices in the treatment of epilepsy is an emerging therapy for those patients whose seizures are not controlled by medications. This article will discuss current treatment options with devices for vagus nerve stimulation, deep brain stimulation and responsive neurostimulation. Emerging therapies in noninvasive neurostimulation such as with trigeminal nerve stimulation, transcranial magnetic stimulation and transcranial direct current stimulation may prove to be promising solutions. Finally, new and enhanced techniques of drug delivery are discussed as well as other devices with potential use in the study and treatment of epilepsy.
\end{abstract}

Medscape: Continuing Medical Education Online

This activity has been planned and implemented in accordance with the Essential Areas andpolicies of the Accreditation Council for Continuing Medical Education through the joint providership of Medscape, LLC and Future Medicine Ltd. Medscape, LLC is accredited by the ACCME to provide continuing medical education for physicians.

Medscape, LLC designates this Journal-based CME activity for a maximum of 1.0 AMAPRA Category 1 Credit $(s)^{\mathrm{TM}}$. Physicians should claim only the credit commensurate with the extent of their participation in the activity.

All other clinicians completing this activity will be issued a certificate of participation. To participate in this journal CME activity: (1) review the learning objectives and author disclosures; (2) study the education content; (3) take the post-test with a $75 \%$ minimum passing score and complete the evaluation at www.medscape.org/journal/fnl; (4) view/print certificate.

\section{RELEASE DATE: 2 DECEMBER 2014; EXPIRATION DATE: 2 DECEMBER 2015}

\section{LEARNING OBJECTIVES}

Upon completion of this activity, participants should be able to:

- Evaluate the benefits and risks associated with use of the vagus nerve stimulator

- Describe responsive neurostimulation in the treatment of epilepsy

- Describe deep brain stimulation in the treatment of epilepsy

- Discuss potential emerging treatments of epilepsy

'Department of Neurology, Dartmouth-Hitchcock Medical Center, Geisel School of Medicine at Dartmouth, One Medical Center Drive, Lebanon, NH 03756, USA

*Author for correspondence: Tel.: +1 603653 6118; Fax: +1 603650 6233; afvanstraten@gmail.com 
Financial \& competing interests disclosure

Editor: Laura Dormer, Editor, Future Science Group.

Disclosure: Laura Dormer has disclosed no relevant financial relationships.

CME author: Charles P Vega, MD, Clinical Professor of Family Medicine, University of California, Irvine.

Disclosure: Charles P Vega MD, served as an advisor or consultant for McNeil Pharmaceuticals.

Authors \& credentials:

Amanda F Van Straten, MD, Department of Neurology, Dartmouth-Hitchcock Medical Center, Lebanon, New Hampshire.

Disclosure: Amanda F Van Straten, $M D$, has disclosed no relevant financial relationships.

Barbara C Jobst, MD, Department of Neurology, Dartmouth-Hitchcock Medical Center, Lebanon, New Hampshire.

Disclosure: Barbara C Jobst, MD, served as an advisor or consultant for NeuroPace Inc. She has received grants for clinical research from NeuroPace Inc., Lundbeck Inc., UCB Inc., Pfizer Inc., Upshire-Smith Inc., the CDC, NIH and the AES. She serves as a part of the scientific advisory board for Neuropace Inc. She is an author for Medlink Inc. as well as an editor for Epilepsia and Epilepsy Current.

No writing assistance was utilized in the production of this manuscript.

\section{KEYWORDS}

- DBS • DCS • devices

- medically intractable epilepsy $\bullet$ RNS $\bullet$ TMS $\bullet$ TNS

- VNS

\section{Indications}

Approximately $30 \%$ of patients with epilepsy remain intractable despite multiple anti-epileptic medication trials $[1,2]$. In these patients, other treatment options such as resective surgery or a disconnection procedure, such as palliative corpus callosotomy, are often necessary. In medically intractable partial epilepsy, no other intervention, including the use of devices is as effective as resective epilepsy surgery in achieving seizure freedom in appropriately selected patients. In a controlled, prospective study of patients with temporal lobe epilepsy, 59\% became seizure free after temporal lobectomy compared with only $8 \%$ in the medical management arm [3]. Retrospective studies show seizure freedom rates between 56 and $83 \%$ in patients with temporal epilepsy following temporal lobectomy [3-6]. Those with mesial temporal onset had better outcomes than those with neocortical onset, and though seizure freedom declined slightly over time, the rate of freedom remained above $55 \%[4,5]$. Seizure freedom rates are similar in nonlesional temporal lobe epilepsy and though less favorable in extratemporal epilepsy with only $13-47 \%$ seizure freedom, surgery often remains the only option for patients after all other nonsurgical options have failed [7-9].

However, epilepsy surgery is not always possible. Patients with generalized seizures, seizures emanating from both hemispheres, and those with a seizure onset zone in eloquent cortex such as motor or language areas are often not surgical candidates. Further, patients that have already undergone respective epilepsy surgery but continue to remain intractable are often poor candidates for further resection. In these patients, more innovative therapies are needed and currently, one such option comes in the form of epilepsy devices. These devices currently include vagus nerve stimulation and responsive brain stimulation, as well as deep brain stimulation. Ongoing studies include trigeminal nerve stimulation, transcranial and transcutaneous stimulation, magnetic stimulation and potential new mechanisms for direct drug delivery to the brain.

\section{Current available devices \\ - Vagus nerve stimulator}

The vagus nerve stimulator (VNS) has been in use since the mid-1990s and is a well-established option in those patients that are unable to undergo surgery. The device is implanted pectorally on the left with leads connecting to the left vagus nerve at the carotid bifurcation. Stimulation is usually initiated 2 weeks after implantation and is delivered in an on-off fashion with the ability to adjust the output current, signal frequency, signal pulse width and signal on and off times. Special magnet-activated stimulus parameters, often with a higher output current, can be triggered with the placement of a magnet briefly over the device by the patient or caregiver. This has been shown to shorten or abort seizures, decrease their intensity and decrease the recovery period [10].

In most practices, the target range of stimulation is between 1.25 and $2.00 \mathrm{~mA}$ with early randomized controlled studies establishing a direct relationship between stimulation intensity and seizure frequency reduction [11]. Changes to signal frequency and pulse width are often manipulated to control side effects and increase battery life [12]. If standard programming is not effective, the option of rapid cycling (term used to describe 
a high duty cycle) is available, though this results in faster depletion of the normal expected battery life of 5-10 years [13,14]. Various mechanisms have been proposed such as increased norepinephrine excretion in the locus coeruleus, increased firing of the serotonergic neurons in the dorsal raphe nucleus, immunomodulation by effecting hippocampal plasticity or by increasing arousal via activation of the reticular activating system [15-17].

There are no head-to-head controlled studies comparing VNS with medication trials. Pre-market trials comparing high and low frequency stimulation in 194 and 114 patients over 12 to 16 weeks found a mean seizure reduction of 27.9 and $24.5 \%$ in the high stimulation group compared with the low stimulation (15.2 and 6.1\%) as well as a $50 \%$ responder rate of 23.4 and $31 \%$ in the high stimulation group compared with 15.7 and $13 \%$ in the low simulation group [18,19]. With long-term use, studies have demonstrated that approximately half of patients will have a greater than $50 \%$ reduction in their seizure frequency after implantation with relatively few (less than 10\%) becoming seizure free $[13,18,20-22]$. These long-term studies are flawed by patient attrition, as the effect of VNS has been shown to increase with time $[18,20,22-23]$. VNS therapy is equally effective in children as demonstrated by a $57.9 \%$ seizure reduction in 128 patients with $64.8 \%$ of those children achieving greater than $50 \%$ reduction during the mean follow-up time of 5.3 years [24]. There have been no studies thus far investigating who are the best responders to VNS, however, those with focal epilepsies seem to do better than those with generalized epilepsies [21].

Overall, VNS therapy is well tolerated. There is a small operative risk with implantation encompassing mainly hemorrhage and infection. Very rarely there can be damage to the carotid artery or laryngeal nerve. The most cited adverse effects with VNS therapy are hoarseness, coughing and dyspnea usually occurring during the 'on' time [21]. Side effects decrease over time [21]. Other less common adverse effects include worsening of sleep apnea as well as dysarthria and/or dysphagia $[16,25]$. Patients and caregivers report increased alertness, shortened seizure duration and increased mood with VNS therapy which can lead to antiepileptic drug medication reduction and to treatment in those with depression [26-31]. The VNS device is MRI compatible using certain coils if the signal frequency is reduced to $0.0 \mathrm{~mA}$ for obtaining brain scans. MRI imaging of the spine cannot be performed with the leads in place.

Transcutaneous activation of the vagus nerve has been utilized and studied via electroacupuncture which has inspired development of transcutaneous vagus nerve stimulator devices [32]. $\mathrm{Nemos}^{\circledR}$, manufactured by Cerbomed (Erlangen, Germany), is a transcutaneous device which stimulates the auricular branch of the vagus nerve and which received the CE mark in Europe with early favorable results in a small cohort study [33]. However, larger studies are still needed. Other transcutaneous devices currently being studied in the treatment of epilepsy include gammaCore ${ }^{\circledR}$ (ElectroCore Medical LLC, NJ, USA), which has been approved for headache treatment in Europe, and Cerbomed's Transcutaneous Vagus Nerve Stimulation $\left(\mathrm{t}-\mathrm{VNS}^{\circledR}{ }^{\circledR}\right.$ [34,35]. Cyberonics (TX, USA) announced early results at the American Epilepsy Society in December 2013 of the E-36 study of their AspireSR ${ }^{\mathrm{TM}}$ generator which combines traditional VNS therapy with seizure detection technology via changes in cardiac rhythms to create a closed-loop, responsive stimulation device [36]. The AspireSRgenerator received the CE mark in Europe in February 2014.

\section{- Responsive neurostimulation}

Responsive neurostimulation (RNS) as a treatment for epilepsy was developed after direct electrical cortical stimulation was shown to terminate after-discharges, a model of epileptiform activity and focal seizures [37]. The RNS ${ }^{\circledR}$ (NeuroPace, CA, USA) is the first closed-loop system developed, tested, and US FDA approved in the treatment of epilepsy. The device is able to detect abnormal electrical activity and then deliver electrical stimulation to the same area as a method of preventing seizures. The RNS was given FDA approval in the USA in November 2013 and is the only responsive stimulation device approved for clinical use.

Treatment with the RNS system is ideal for the patient with either focal epilepsy that is otherwise unable to undergo epilepsy surgery or the patient with multifocal epilepsy with no more than two discrete epileptic onset zones. Up to two leads containing four electrodes each, utilizing implantable depth and/or cortical strip electrodes, can be placed into/over the epileptogenic zone (Figure 1). The leads are connected to the device which is implanted in the skull. The device continuously records and processes intracranial EEG data obtained from the placed leads and 
stores up to $30 \mathrm{~min}$ of EEG activity. The patient is required to interrogate the device with a remote monitor frequently and more importantly, after seizures. The data are transferred online to a secure patient data management system. The data can be reviewed and monitored by the physician and seizure detection algorithms can be tested. This data are utilized to program the device to the individual patient's seizure onset pattern. When the device detects a seizure, it is programmed to deliver up to five individual pulses of stimulation. The stimulation depends on seizure onset location and electrode configuration. The signal pulse width is set between 160 and $200 \mu$ s with the signal frequency and duration set at $100-200 \mathrm{~Hz}$ and $100 \mathrm{~ms}$, respectively. The expected battery life is between 2.5 and 4 years [38].

In the RNS System Pivotal Clinical Investigation, a total of 191 patients were studied in a randomized control trial with a blinded period of 12 weeks followed by an open-label period of 84 weeks [39]. The seizure reduction
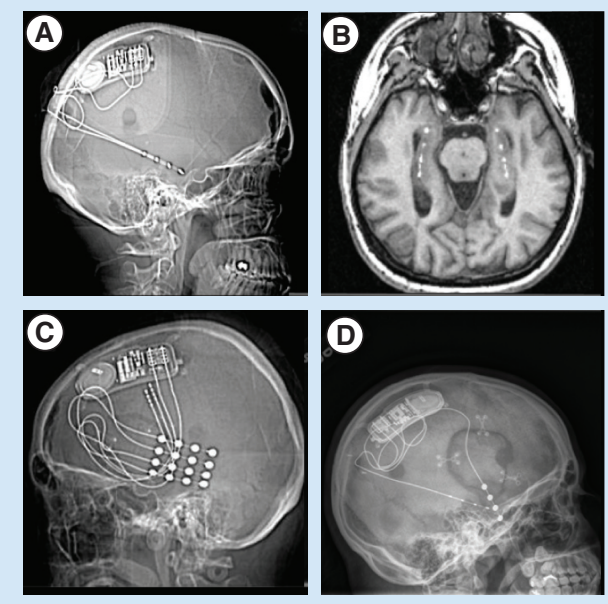

Figure 1. Different implantations for RNS ${ }^{\circledR}$.

(A \& B) Implantation of depth electrodes in the bilateral hippocampi demonstrated by CT scan of the head on the left and axial T2 FLAIR MRI on the right. On the CT scan, you can also see the RNS (responsive neurostimulation) recording and stimulation device that is implanted in the skull. (C) CT scan showing implantation using grid electrodes for neocortical stimulation of the left lateral temporal language area. Only eight contacts on two leads are connected. (D) CT scan showing implantation of a depth electrode in the hippocampus and an electrode strip over the lateral temporal neocortex. over the entire blinded observation period was $37.9 \%$ in the treatment group versus $17.3 \%$ in the control group $(\mathrm{p}=0.012)$ with overall seizure reduction at the end of the 3-month blinded period of $41.5 \%$ in the treatment arm and $9.4 \%$ in the control $(\mathrm{p}=0.008)$. However, there was no difference between the groups in percentage of patients that achieved a greater than $50 \%$ reduction in seizure frequency [39]. Long-term responses increased with prolonged use as seen with VNS. At 5 years, median seizure reduction was greater than $65 \%$ with approximately $15 \%$ of patients achieving seizure freedom $[35,36]$. A 2-year followup study found no difference between patients with mesial temporal lobe epilepsy and nonmesial temporal lobe epilepsy (mean seizure reduction of 55 and $58 \%$, respectively) but that patients with a single seizure focus did better overall all than those with multifocal epilepsy [40].

RNS is well tolerated with low major operative risks including nonclinical serious intracranial hemorrhage (2.1\%) and infection (5.2\%). More common adverse symptoms included headache and pain at the implantation site [39]. There were no adverse effects on mood inventories or neuropsychological measures at the end of the blinded period or at 2 years out. In fact, there was an actual improvement in verbal functioning, visuospatial ability and memory at both 1 and 2 years follow-up in the RNS patients [39]. MRI of the brain with the RNS system in place is not advisable as there is intense artifact from the device and further, there is a theoretical risk for lead heating.

\section{- Deep brain stimulation}

Deep brain stimulation (DBS) has been studied and explored for many years. The thalamus has been a popular site of stimulation due to its connections to the limbic system and role in initiation of generalized seizures and propagation of partial seizures [41,42]. Besides the thalamus, other targeted areas have included the caudate nucleus [43], the subthalamic nucleus [44], the locus coeruleus [45], the cerebellum [46-49] and the hippocampus [50,51]. Cerebellar stimulation has not been effective, but hippocampal stimulation has been promising in early studies [50-52]. The CoRaStiR trial is ongoing and compares medial temporal lobe resection with amygdala/hippocampal stimulation and sham stimulation [53].

Thalamic targets for stimulation have included the anterior nucleus, the centromedian 
nucleus [54], the mediodorsal nucleus [55] and the ventroposterolateral nucleus [56]. Stimulation in the centromedian nucleus was initially thought to be effective in epilepsy. In early uncontrolled studies, seizure reduction was measured between 80 and $100 \%$ with the additional benefit of normalization of the EEG and improvements in psychological outcomes [54]. However, when these results were followed up with a doubleblind pilot study, seizure reduction rates were measured at only $30 \%$ without statistical significance between the stimulated group and the control group [57].

Besides various cohort studies, there has been only a single randomized controlled trial with sufficient sample size for thalamic stimulation in epilepsy. The SANTE trial randomized 110 patients into a stimulation group versus a sham group. Implantation utilized the same DBS device that is used in Parkinson's disease with the following settings: $5 \mathrm{~V}$ amplitude, $90 \mu$ s pulse width, $140 \mathrm{~Hz}$ frequency and $1 \mathrm{~min}$ on $/ 5$ min off targeted at the anterior nucleus (Figure 2). Median seizure reduction at the end of the 3-month observation period was $29 \%$ in the stimulation group compared with $17 \%$ in the control group [58]. This was considered statistically significant using a generalized estimating equation $(\mathrm{p}=0.038)$. However, as with the other neurostimulation modalities, improvement was seen over the long term. At the end of 3 months, median seizure reduction was $40.5 \%$ in the stimulated group compared with $14.5 \%$ in the control group [58]. At 1-, 2- and 5 -year follow-up, the efficacy improved further with a median seizure reduction of $69 \%$ and $16 \%$ of patients achieving seizure freedom [59]. Further, those patients with temporal lobe epilepsy seemed to respond better than patients with extratemporal or neocortical epilepsy [58]. A follow-up study looking at recruitment rhythm comparisons between responders and nonresponders was undertaken, but no results have been generated as yet (SANTE-RR) [60]. Over the long term, approximately $16 \%$ of patients withdrew from the study. There were no symptomatic intracranial hemorrhages but $4.5 \%$ of patients had hemorrhages detected by imaging. The most common serious side effect was infection, either at the generator or lead site, which occurred in $12.7 \%$ of patients. These were all successfully treated. Finally, patients in the stimulated group reported more cognitive and depressive symptoms, though this was

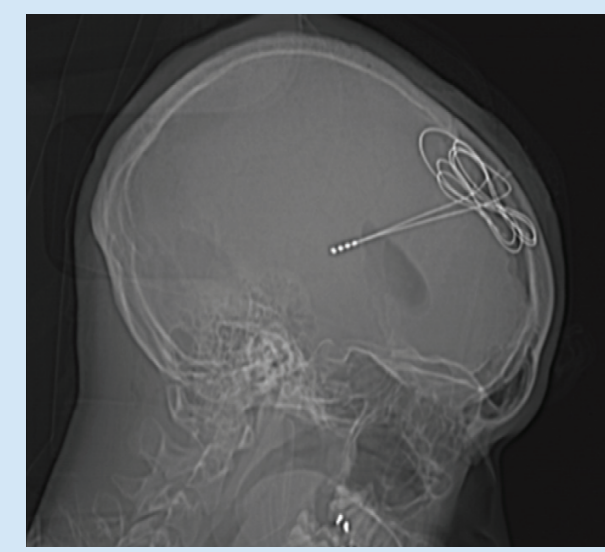

Figure 2. Placement of deep brain stimulation electrodes in the anterior nucleus of the thalamus.

not statistically significant. The average battery life was around 3 years and the device is not compatible with MRI [58]. The device has the CE mark in Europe but was not approved by the FDA in the USA when presented in 2010. There were concerns at that time that the 3-month blinded-period of the SANTE trial was too short to adequately study the device. Now that longterm use data continues to show positive results in the treatment of epilepsy, we are hopeful that DBS will become available in the USA.

\section{Noninvasive stimulation: an emerging practice}

Neurostimulation is an established treatment of epilepsy and has shown to be effective. It opens up an additional treatment venue to patients who are not candidates for epilepsy surgery. Emerging therapies are focused on other modes of stimulation and especially noninvasive stimulation. There are several models of noninvasive vagus nerve stimulators as mentioned earlier. Other noninvasive neurostimulation devices include transcutaneous trigeminal nerve stimulation (tTNS or TNS), transcranial magnetic stimulation (TMS) and transcranial direct current stimulation ( $\mathrm{tDCS}$ ).

NeuroSigma (CA, USA) has manufactured the Monarch ${ }^{\mathrm{TM}}$ eTNSTM, which utilizes an external removable single gel electrode applied to the forehead to transcutaneously stimulate the trigeminal nerve. The device is recommended to be worn a minimum of $12 \mathrm{~h}$ per day, or overnight while sleeping, and delivers an on/off stimulation pattern similar to VNS to the 
bilateral orbital and supratrochlear branches of the trigeminal nerve. The theorized mechanism involves activation of the ascending reticular activating system through sensory nerve input (via the trigeminal nerve in this case) to cause desynchronization by way of widespread cortical projections [61]. Tentatively, any sensory nerve could be used under this mechanism barring autonomic disruption. The Phase II study with 50 patients treated over 18 weeks was recently completed using the following parameters: $2 \mathrm{~Hz}$ stimulation frequency, $2 \mathrm{~s}$ on and $90 \mathrm{~s}$ off duty cycle with a pulse width of $50 \mu$ s. Median seizure reduction over the entire 18 -week period was $16.1 \%$ reduction in the treatment group and $8.0 \%$ in the control group with $30.2 \%$ of patients in the treatment group achieving greater than $50 \%$ reduction compared with $21.1 \%$ in the control group [62]. This was not clinically significant. However, at the end of the 18 -week period, there was a significant difference in the $50 \%$ responder rate of $40.5 \%$ in the treatment group compared with $15.6 \%$ in the control group ( $p=0.078$ ) [62]. Longer followup may reveal further widening between the two groups. Overall, the eTNS system is well tolerated with only minor side effects including anxiety, headache and skin irritation as the most common finding [63]. Further, there was a significant improvement in mood as measured with the Beck Depression Inventory in the treatment group [62]. The major advantage with TNS is that it is external, does not require surgical placement and is easily discontinued if intolerable or ineffectual. A Phase III study is currently being planned which will hopefully give us more information on this emerging therapy [64].

Repetitive TMS (rTMS or TMS) has been studied for over 20 years and functions via an electric coil placed over the scalp using rapidly changing magnetic fields to induce current changes in the brain [65]. It has been shown to reduce cortical excitability and reduce interictal epileptiform activities [66]. Animal models showed that TMS blocked seizures from occurring [67]. Human studies have had mixed results. Initially, TMS was being applied at the vertex without individualization for seizure onset zone. These studies showed negligible changes in seizure frequency with results being minimal and brief [68-70]. More recent studies have focused on patients with focal and multifocal epilepsies with TMS administered focally to each individual's epileptogenic zones which has produced better results [68,71-74]. A randomized controlled trial of 21 patients with cortical dysplasias underwent localized TMS or sham stimulation with follow-up at 60 days. The treatment group had 58\% mean seizure reduction compared with no significant change in the sham group $(p<0.0001)$. Eight-three percent of the treatment group achieved a greater than $50 \%$ reduction in mean seizure frequency while none of the nine patients in the sham group did [74]. There are two active studies currently recruiting for multifocal and unifocal refractory epilepsy. TMS may also improve mood [75]. Effects on cognition are mixed and likely associated with the mood effects [76].

tDCS works by direct electrical stimulation on the scalp over the area of interest via electrodes which can in turn induce either hyperexcitability or hypoexcitability of the cortex depending on the type of stimulation. This type of stimulation has been studied and used regularly in neurorehabilitation and is being studied in psychiatric disorders. Cathodal stimulation of the epileptogenic zone to decrease neuronal excitability has only been recently studied in epilepsy patients. A randomized control trial of 19 patients with medically refractory focal and multifocal epilepsy who underwent a single 20 -min session of tDCS found a reduction in interictal epileptiform discharges and had an approximate $40 \%$ reduction in mean seizure frequency at 1-month follow-up compared with no change in the sham stimulation group [77]. Further studies in patients with refractory focal onset seizures are ongoing [78].

\section{Drug delivery devices}

Another emerging therapy using devices is aimed at enhanced delivery of antiepileptic medications. Intrathecal and intraventricular drug delivery bypass the blood-brain barrier and thus enhance pharmacological activity in the brain as well as limit systemic effects of administered medications [79]. Transmeningeal delivery of antiepileptic agents requires a constant infusion and would be most effective in neocortical epilepsies [80,81]. All of these methods, however, are still untargeted in that normal brain tissue is still exposed to the antiepileptic agent. More targeted methods include direct intracerebral delivery of therapeutic agents via a catheter placed directly into the epileptic zone [82-84]. This method is limited by a nonuniform diffusion of therapeutic agents which reduces control of the therapy. A possible solution has arisen in convection-enhanced 
delivery. This process administers drug by slow infusion under positive pressure and results in a larger volume of distribution as well as a more uniform distribution of the therapeutic agent then what is achieved by diffusion methods. Additionally, with convection-enhanced delivery, the target of drug delivery has a sharply defined border which results in more refined focalized treatment [85]. Early animal studies have been promising $[86,87]$. Finally, there have been several animal studies dedicated to the production of drug-eluding wafers that could be implanted intracranially at the site of epileptogenesis [88,89]. This method provides the same benefits as with the previous methods but would be limited in the need for repeat craniotomies to replenish the wafer. There are no active human clinical trials involving any of these methods at this time.

\section{Other devices}

These nonstimulation, nonpharmacological devices provide yet another mechanism for epilepsy treatment. Cold water applied directly to the brain in the operating room and induced hypothermia in comatose patients have both been shown to stop seizures [90]. In animal models, focal brain cooling has demonstrated an inhibition in epileptic kindling as well as an increased threshold for chemically induced seizures [91,92]. Focal, passive cooling in rats after traumatic brain injury as little as $2^{\circ} \mathrm{C}$ has been shown to prevent chronic recurrent seizures [93]. The development of an external device to cool the head and neck and thereby the brain is currently being studied as well as implantable devices for focal cooling in patients with focal epilepsy [94]. Other ideas evolve around the use of a partial re-breather wherein the theory is that the induced respiratory acidosis by re-breathing exhaled gases would cause a drop in systemic $\mathrm{pH}$ that would stop or prevent seizure activity [95].

Other epilepsy devices have aimed at seizure detection/alerting systems. These would be especially important in the study and prevention of sudden unexpected death in epilepsy (SUDEP). These devices employ monitoring of electromyography data, eye and muscle movements, and body vibrations with the use of electrodes, accelerometers, and bed alarms to name a few $[96,97]$. NeuroVista (WA, USA) had completed early human studies on their implanted wireless device that continuously records and analyzes intracranial EEG data, however, the company is no longer active [98]. Through all of these methods, the acquired data are being used to design computer models that could potentially predict seizures. Seizure prediction would be invaluable to the patient but also to understanding of seizure behavior and pathogenesis. If seizures could be adequately predicted, treatments and lifestyle could be tailored to seizure occurrence.

\section{Conclusion}

The use of devices in the treatment of epilepsy is a growing field. Currently, only two devices (VNS and RNS) are approved for use in the USA and while deep brain stimulation has not been approved in the USA it had been approved for treatment of epilepsy in Europe and over 30 different countries. While epilepsy surgery remains the mainstay of treatment for medically intractable epilepsy, this may not always be true in the future. The use of devices avoids the functional deficits of epilepsy surgery, minimizes the need for antiepileptic medications and their potential side effects and at the same time is more inclusive to all epilepsy types, such as generalized epilepsies, focal epilepsies involving eloquent cortex or even patients who previously had epilepsy surgery but continue to have intractable seizures. The development of devices to treat epilepsy is difficult, especially in neurostimulation. It is clear that current animal models are not representative [54]. Additionally, the number of variables included with stimulation, in other words, pulse width, frequency and duty cycle, among others, can be overwhelming. Performing blinded studies can be difficult and costly.

With few current options, the use of devices for the treatment of epilepsy has been limited. However, with the recent FDA approval of RNS and with so many more devices on the horizon, the use of devices in treatment of epilepsy is likely to become much more prominent.

\section{Future perspective}

This article outlines currently available devices used in the treatment of epilepsy as well as devices that are currently in development. We predict that in the next several years, DBS will be approved for use in the USA, the use of RNS will be well established, and at the same time, transcutaneous TNS should be introduced to the market. While several different devices are under development, convection-enhanced drug delivery seems especially promising. 


\section{EXECUTIVE SUMMARY}

\section{Currently available devices}

- Vagus nerve stimulator, responsive nerve stimulator and deep brain stimulator.

\section{Devices in development}

- Neurostimulation: trigeminal nerve stimulation, transcranial magnetic stimulation and transcranial direct current stimulation.

- Drug delivery: convection-enhanced delivery.

- Other: cooling devices, seizure detection and seizure alarm systems.

\section{Future studies involving devices}

- Improved closed loop systems that detect and treat seizures without external intervention.

- Better animal models.

- Longer studies.

- Better understanding of device stimulation parameters.

\section{References}

Papers of special note have been highlighted as:

- of interest; $\bullet$ of considerable interest

1 Kwan P, Brodie MJ. Effectiveness of first antiepileptic drug. Epilepsia 42(10), 1255-1260 (2001).

-• Classic, frequently cited article demonstrating the effect of oral antiepileptic medications in newly diagnosed patients.

2 Mattson RH, Cramer JA, Collins JF. A comparison of valproate with carbamazepine for the treatment of complex partial seizures and secondarily generalized tonic-clonic seizures in adults. The Department of Veterans Affairs Epilepsy Cooperative Study No. 264 Group. N. Engl. J. Med. 327(11), 765-771 (1992).

3 Wiebe S, Blume WT, Girvin JP, Eliasziw M. A randomized, controlled trial of surgery for temporal-lobe epilepsy. N. Engl. J. Med. 345(5), 311-318 (2001).

4 Spencer SS, Berg AT, Vickrey BG et al. Initial outcomes in the Multicenter Study of Epilepsy Surgery. Neurology 61(12), 1680-1685 (2003).

5 Yoon HH, Kwon HL, Mattson RH, Spencer DD, Spencer SS. Long-term seizure outcome in patients initially seizure-free after resective epilepsy surgery. Neurology 61(4), 445-450 (2003).

6 Engel J Jr, McDermott MP, Wiebe S et al. Early surgical therapy for drug-resistant temporal lobe epilepsy: a randomized trial. JAMA 307(9), 922-930 (2012).
7 Lazow SP, Thadani VM, Gilbert KL et al. Outcome of frontal lobe epilepsy surgery. Epilepsia 53(10), 1746-1755 (2012).

8 Mosewich RK, So EL, O’Brien TJ et al. Factors predictive of the outcome of frontal lobe epilepsy surgery. Epilepsia 41(7), 843-849 (2000).

9 Simasathien T, Vadera S, Najm I, Gupta A, Bingaman W, Jehi L. Improved outcomes with earlier surgery for intractable frontal lobe epilepsy. Ann. Neurol. 73(5), 646-654 (2013).

10 Hammond EJ, Uthman BM, Reid SA, Wilder BJ. Electrophysiological studies of cervical vagus nerve stimulation in humans: I. EEG effects. Epilepsia 33(6), 1013-1020 (1992).

11 Heck C, Helmers SL, Degiorgio CM. Vagus nerve stimulation therapy, epilepsy, and device parameters: scientific basis and recommendations for use. Neurology 59(6 Suppl. 4), S31-S37 (2002).

12 Liporace J, Hucko D, Morrow R et al. Vagal nerve stimulation: adjustments to reduce painful side effects. Neurology. 57(5), 885-886 (2001).

13 Degiorgio CM, Thompson J, Lewis $\mathrm{P}$ et al. Vagus nerve stimulation: analysis of device parameters in 154 patients during the long-term XE5 study. Epilepsia 42(8), 1017-1020 (2001).

14 Degiorgio C, Heck C, Bunch S et al. Vagus nerve stimulation for epilepsy: randomized comparison of three stimulation paradigms. Neurology 65(2), 317-319 (2005).
15 Aalbers M, Vles J, Klinkenberg S, Hoogland G, Majoie M, Rijkers K. Animal models for vagus nerve stimulation in epilepsy. Exp. Neurol. 230(2), 167-175 (2011).

16 Rutecki P. Anatomical, physiological, and theoretical basis for the antiepileptic effect of vagus nerve stimulation. Epilepsia 31(Suppl 2), S1-S6 (1990).

17 Schachter SC, Wheless JW. The evolving place of vagus nerve stimulation therapy. Neurology. 59(6 Suppl. 4), S1-S2 (2002).

18 Handforth A, Degiorgio CM, Schachter SC et al. Vagus nerve stimulation therapy for partial-onset seizures: a randomized active-control trial. Neurology 51(1), 48-55 (1998).

19 A randomized controlled trial of chronic vagus nerve stimulation for treatment of medically intractable seizures. The Vagus Nerve Stimulation Study Group. Neurology 45(2), 224-230 (1995).

20 Ben-Menachem E, Manon-Espaillat R, Ristanovic R et al. Vagus nerve stimulation for treatment of partial seizures: 1. A controlled study of effect on seizures. First International Vagus Nerve Stimulation Study Group. Epilepsia 35(3), 616-626 (1994).

21 Elliott RE, Morsi A, Kalhorn SP et al. Vagus nerve stimulation in 436 consecutive patients with treatment-resistant epilepsy: long-term outcomes and predictors of response. Epilepsy Behav. 20(1), 57-63 (2011).

- Important study looking at the long-term effectiveness of the vagus nerve stimulator. 
22 Elliott RE, Morsi A, Tanweer O et al. Efficacy of vagus nerve stimulation over time: review of 65 consecutive patients with treatment-resistant epilepsy treated with VNS $>10$ years. Epilepsy Behav. 20(3), 478-483 (2011).

23 Morris GL 3rd, Mueller WM. Long-term treatment with vagus nerve stimulation in patients with refractory epilepsy. The Vagus Nerve Stimulation Study Group E01-E05. Neurology 53(8), 1731-1735 (1999).

24 Elliott RE, Rodgers SD, Bassani L et al. Vagus nerve stimulation for children with treatment-resistant epilepsy: a consecutive series of 141 cases. J. Neurosurg. Pediatr. 7(5), 491-500 (2011).

25 Marzec M, Edwards J, Sagher O, Fromes G, Malow BA. Effects of vagus nerve stimulation on sleep-related breathing in epilepsy patients. Epilepsia 44(7), 930-935 (2003).

26 Rush AJ, Marangell LB, Sackeim HA et al. Vagus nerve stimulation for treatmentresistant depression: a randomized, controlled acute phase trial. Biol. Psychiatry 58(5), 347-354 (2005)

27 Klinkenberg S, Majoie HJ, Van Der Heijden MM, Rijkers K, Leenen L, Aldenkamp AP. Vagus nerve stimulation has a positive effect on mood in patients with refractory epilepsy. Clin. Neurol. Neurosurg. 114(4), 336-340 (2012).

28 Malow BA, Edwards J, Marzec M, Sagher O, Ross D, Fromes G. Vagus nerve stimulation reduces daytime sleepiness in epilepsy patients. Neurology 57(5), 879-884 (2001).

29 Marangell LB, Rush AJ, George MS et al. Vagus nerve stimulation (VNS) for major depressive episodes: one year outcomes. Biol. Psychiatry 51(4), 280-287 (2002)

30 George MS, Rush AJ, Marangell LB et al. A one-year comparison of vagus nerve stimulation with treatment as usual for treatment-resistant depression. Biol. Psychiatry 58(5), 364-373 (2005)

31 Dodrill CB, Morris GL. Effects of vagal nerve stimulation on cognition and quality of life in epilepsy. Epilepsy Behav. 2(1), 46-53 (2001).

32 Cakmak YO. Epilepsy, electroacupuncture and the nucleus of the solitary tract. Acupunct. Med. 24(4), 164-168 (2006).

33 Stefan H, Kreiselmeyer G, Kerling F et al. Transcutaneous vagus nerve stimulation (t-VNS) in pharmacoresistant epilepsies: a proof of concept trial. Epilepsia 53(7), e115-e118 (2012)

34 ElectroCore LLC. Neurostimulation to the vagus nerve for the reduction in frequency of seizures associated with epilepsy.

http://clinicaltrials.gov

35 Cerbomed. Transcutaneous non-invasive stimulation of the vagus nerve for the treatment of difficult-to-treat epilepsy. http://clinicaltrials.gov

36 Cyberonics, Inc. Seizure Detection and Automatic Magnet Mode Performance Study (E-36). http://clinicaltrials.gov

37 Lesser RP, Kim SH, Beyderman L et al. Brief bursts of pulse stimulation terminate afterdischarges caused by cortical stimulation. Neurology 53(9), 2073-2081 (1999).

38 Neuropace. RNS System User Manual. www.neuropace.com/product/pdfs

39 Morrell MJ. Responsive cortical stimulation for the treatment of medically intractable partial epilepsy. Neurology 77(13), 1295-1304 (2011).

-• Important first study showing the results of responsive brain stimulation.

40 Heck CN, King-Stephens D, Massey AD et al. Two-year seizure reduction in adults with medically intractable partial onset epilepsy treated with responsive neurostimulation: final results of the RNS System Pivotal trial. Epilepsia 55(3), 432-441 (2014).

41 Schwartzkroin PA. Origins of the epileptic state. Epilepsia 38(8), 853-858 (1997).

42 Engel J, Pedley TA. Epilepsy: a Comprehensive Textbook (2nd Edition). Wolters Kluwer Health/Lippincott Williams \& Wilkins, Philadelphia, PA, USA (2008).

- Great reference textbook on everything to do with epilepsy.

43 Chkhenkeli SA, Chkhenkeli IS. Effects of therapeutic stimulation of nucleus caudatus on epileptic electrical activity of brain in patients with intractable epilepsy. Stereotact. Funct. Neurosurg. 69 (1-4 Pt 2), 221-224 (1997).

44 Handforth A, Desalles AA, Krahl SE. Deep brain stimulation of the subthalamic nucleus as adjunct treatment for refractory epilepsy. Epilepsia 47(7), 1239-1241 (2006).

45 Feinstein B, Gleason CA, Libet B. Stimulation of locus coeruleus in man. Preliminary trials for spasticity and epilepsy. Stereotact. Funct. Neurosurg. 52(1), 26-41 (1989).

46 Cooper IS, Amin I, Gilman S. The effect of chronic cerebellar stimulation upon epilepsy in man. Trans. Am. Neurol. Assoc. 98 192-196 (1973).

47 Cooper IS, Amin I, Riklan M, Waltz JM, Poon TP. Chronic cerebellar stimulation in epilepsy. Clinical and anatomical studies. Arch. Neurol. 33(8), 559-570 (1976).

48 Davis R, Barolat-Romana G, Engle H. Chronic cerebellar stimulation for cerebral palsy-five-year study. Acta Neurochir. Suppl. (Wien). 30, 317-332 (1980).

49 Davis R. Cerebellar stimulation for cerebral palsy spasticity, function, and seizures. Arch. Med. Res. 31(3), 290-299 (2000).

50 Boon P, Vonck K, De Herdt V et al. Deep brain stimulation in patients with refractory temporal lobe epilepsy. Epilepsia 48(8), 1551-1560 (2007).

51 Velasco AL, Velasco F, Velasco M, Trejo D, Castro G, Carrillo-Ruiz JD. Electrical stimulation of the hippocampal epileptic foci for seizure control: a double-blind, long-term follow-up study. Epilepsia 48(10), 1895-1903 (2007).

52 Wright GD, Mclellan DL, Brice JG. A double-blind trial of chronic cerebellar stimulation in twelve patients with severe epilepsy. J. Neurol. Neurosurg. Psychiatry. 47(8), 769-774 (1984).

53 University Hospital, Ghent. Controlled Randomized Stimulation Versus Resection (CoRaStiR). http://clinicaltrials.gov

54 Velasco M, Velasco F, Velasco AL et al. Electrocortical and behavioral responses produced by acute electrical stimulation of the human centromedian thalamic nucleus. Electroencephalogr. Clin. Neurophysiol. 102(6), 461-471 (1997)

55 Takase K, Shigeto H, Kamada T, Ohyagi Y, Kira J. Efficacy of mediodorsal thalamic nucleus stimulation in a rat model of cortical seizure. Fukuoka Igaku Zasshi 100 (8), 274-280 (2009).

56 Mondragon S, Lamarche M. Suppression of motor seizures after specific thalamotomy in chronic epileptic monkeys. Epilepsy Res. 5(2), 137-145 (1990).

57 Fisher RS, Uematsu S, Krauss GL et al. Placebo-controlled pilot study of centromedian thalamic stimulation in treatment of intractable seizures. Epilepsia 33(5), 841-851 (1992).

58 Fisher R, Salanova V, Witt $\mathrm{T}$ et al. Electrical stimulation of the anterior nucleus of thalamus for treatment of refractory epilepsy. Epilepsia 51(5), 899-908 (2010).

- $\quad$ The SANTE study, which is the only randomized controlled trial of sufficient size in deep brain stimulation in epilepsy.

59 Salanova V, Fisher R, Sante G. Long term efficacy of the SANTE trial (Stimulation of the Anterior Nuclues of Thalamus for 
Epilepsy). Presented at: 66th Annual American Epilepsy Society Annual Meeting. San Diego, CA, USA, 30 November-4 December 2012.

60 MedtronicNeuro. Stimulation of the Anterior Nucleus of the Thalamus for Epilepsy Recruitment Stimulation of the Anterior Nucleus of the Thalamus for Epilepsy Recruitment Rhythm (SANTE RR). http://clinicaltrials.gov

61 Fanselow EE. Central mechanisms of cranial nerve stimulation for epilepsy. Surg. Neurol. Int. 3(Suppl. 4), S247-S254 (2012).

62 Degiorgio CM, Soss J, Cook IA et al. Randomized controlled trial of trigeminal nerve stimulation for drug-resistant epilepsy. Neurology 80 (9), 786-791 (2013).

- Important early study looking at the results of trigeminal nerve stimulation in epilepsy.

63 Pop J, Murray D, Markovic D,

Degiorgio CM. Acute and long-term safety of external trigeminal nerve stimulation for drug-resistant epilepsy. Epilepsy Behav. 22(3), 574-576 (2011).

64 Neurosigma, Inc. External Trigeminal Nerve Stimulation for Drug Resistent Epilepsy (eTNS for DRE).

http://clinicaltrials.gov

65 Barker AT, Jalinous R, Freeston IL. Noninvasive magnetic stimulation of human motor cortex. Lancet 1(8437), 1106-1107 (1985).

66 Tergau F, Naumann U, Paulus W, Steinhoff BJ. Low-frequency repetitive transcranial magnetic stimulation improves intractable epilepsy. Lancet 353(9171), 2209 (1999).

67 Weiss SR, Li XL, Rosen JB, Li H, Heynen T, Post RM. Quenching: inhibition of development and expression of amygdala kindled seizures with low frequency stimulation. Neuroreport 6(16), 2171-2176 (1995).

68 Brasil-Neto JP, De Araujo DP, Teixeira WA, Araujo VP, Boechat-Barros R. Experimental therapy of epilepsy with transcranial magnetic stimulation: lack of additional benefit with prolonged treatment. Arq Neuropsiquiatr. 62(1), 21-25 (2004).

69 Cantello R, Rossi S, Varrasi C et al. Slow repetitive TMS for drug-resistant epilepsy: clinical and EEG findings of a placebocontrolled trial. Epilepsia 48(2), 366-374 (2007).

70 Theodore WH, Hunter K, Chen R et al. Transcranial magnetic stimulation for the treatment of seizures: a controlled study. Neurology 59(4), 560-562 (2002).
71 Sun W, Mao W, Meng X et al. Low-frequency repetitive transcranial magnetic stimulation for the treatment of refractory partial epilepsy: a controlled clinical study. Epilepsia 53(10), 1782-1789 (2012).

72 Santiago-Rodriguez E, Cardenas-Morales L, Harmony T, Fernandez-Bouzas A, Porras-Kattz E, Hernandez A. Repetitive transcranial magnetic stimulation decreases the number of seizures in patients with focal neocortical epilepsy. Seizure 17(8), 677-683 (2008).

73 Joo EY, Han SJ, Chung SH, Cho JW, Seo DW, Hong SB. Antiepileptic effects of low-frequency repetitive transcranial magnetic stimulation by different stimulation durations and locations. Clin. Neurophysiol. 118(3), 702-708 (2007).

74 Fregni F, Otachi PT, Do Valle A et al. A randomized clinical trial of repetitive transcranial magnetic stimulation in patients with refractory epilepsy. Ann. Neurol. 60(4), 447-455 (2006)

- Early study evaluating transcranial magnetic stimulation in the treatment of epilepsy.

75 Boggio PS, Fregni F, Bermpohl F et al. Effect of repetitive TMS and fluoxetine on cognitive function in patients with Parkinson's disease and concurrent depression. Mov. Disord. 20(9), 1178-1184 (2005).

76 Bermpohl F, Fregni F, Boggio PS et al. Effect of low-frequency transcranial magnetic stimulation on an affective go/no-go task in patients with major depression: role of stimulation site and depression severity. Psychiatry Res. 141(1), 1-13 (2006).

77 Fregni F, Thome-Souza S, Nitsche MA, Freedman SD, Valente KD, Pascual-Leone A. A controlled clinical trial of cathodal DC polarization in patients with refractory epilepsy. Epilepsia 47(2), 335-342 (2006).

- Early study evaluating direct current stimulation in the treatment of epilepsy.

78 Suarez MV. A controlled clinical trial of cathodal transcranial direct current stimulation in patients with refractory epilepsy.

http://clinicaltrials.gov

79 Barcia JA, Gallego JM. Intraventricular and intracerebral delivery of anti-epileptic drugs in the kindling model. Neurotherapeutics 6(2), 337-343 (2009).

80 Ludvig N, Baptiste SL, Tang HM et al. Localized transmeningeal muscimol prevents neocortical seizures in rats and nonhuman primates: therapeutic implications. Epilepsia 50(4), 678-693 (2009).
81 John JE, Baptiste SL, Sheffield LG et al. Transmeningeal delivery of GABA to control neocortical seizures in rats. Epilepsy Res. 75(1), 10-17 (2007).

82 Stein AG, Eder HG, Blum DE, Drachev A, Fisher RS. An automated drug delivery system for focal epilepsy. Epilepsy Res. 39(2), 103-114 (2000).

83 Anschel DJ, Ortega E, Fisher RS. Diazepam prophylaxis for bicuculline-induced seizures: a rat dose-response model. Neurosci. Lett. 356(1), 66-68 (2004).

84 Anschel DJ, Ortega EL, Kraus AC, Fisher RS. Focally injected adenosine prevents seizures in the rat. Exp. Neurol. 190 (2), 544-547 (2004).

85 Rogawski MA. Convection-enhanced delivery in the treatment of epilepsy. Neurotherapeutics 6(2), 344-351 (2009).

86 Gasior M, White NA, Rogawski MA. Prolonged attenuation of amygdala-kindled seizure measures in rats by convectionenhanced delivery of the $\mathrm{N}$-type calcium channel antagonists omega-conotoxin GVIA and omega-conotoxin MVIIA. J. Pharmacol. Exp. Ther. 323(2), 458-468 (2007).

87 Gasior M, Tang R, Rogawski MA. Longlasting attenuation of amygdala-kindled seizures after convection-enhanced delivery of botulinum neurotoxins a and B into the amygdala in rats. J. Pharmacol. Exp. Ther. 346(3), 528-534 (2013).

88 Kubek MJ, Liang D, Byrd KE, Domb AJ. Prolonged seizure suppression by a single implantable polymeric-TRH microdisk preparation. Brain Res. 809(2), 189-197 (1998).

89 Tamargo RJ, Rossell LA, Kossoff EH, Tyler BM, Ewend MG, Aryanpur JJ. The intracerebral administration of phenytoin using controlled-release polymers reduces experimental seizures in rats. Epilepsy Res. 48(3), 145-155 (2002).

90 Rothman SM. The therapeutic potential of focal cooling for neocortical epilepsy. Neurotherapeutics 6(2), 251-257 (2009).

91 Burton JM, Peebles GA, Binder DK, Rothman SM, Smyth MD. Transcortical cooling inhibits hippocampal-kindled seizures in the rat. Epilepsia 46(12), 1881-1887 (2005).

92 Atkins CM, Truettner JS, Lotocki G et al. Post-traumatic seizure susceptibility is attenuated by hypothermia therapy. Eur. J. Neurosci. 32(11), 1912-1920 (2010).

93 D'ambrosio R, Eastman CL, Darvas F et al. Mild passive focal cooling prevents epileptic seizures after head injury in rats. Ann. Neurol. 73(2), 199-209 (2013). 
94 NINDS. Reducing Seizure Frequency Using Cooling of the Head and Neck.

http://clinicaltrials.gov

95 University of Aarhus. Stopping and Preventing Epileptic Seizures Using a Partial Rebreathing Mask (EpiCapno).

http://clinicaltrials.gov
3-Dimensional Accelerometer Sub-Study. http://clinicaltrials.gov

97 Brain Sentinel. Seizure Detection and Warning System for Epilepsy Patients. http://clinicaltrials.gov
98 Cook MJ, O’Brien TJ, Berkovic SF et al. Prediction of seizure likelihood with a long-term, implanted seizure advisory system in patients with drug-resistant epilepsy: a first-in-man study. Lancet Neurol. 12(6), 563-571 (2013). 


\section{Future of epilepsy treatment: integration of devices}

To obtain credit, you should first read the journal article. After reading the article, you should be able to answer the following, related, multiplechoice questions. To complete the questions (with a minimum $75 \%$ passing score) and earn continuing medical education (CME) credit, please go to www.medscape.org/journal/fnl. Credit cannot be obtained for tests completed on paper, although you may use the worksheet below to keep a record of your answers. You must be a registered user on Medscape.org. If you are not registered onMedscape.org, please click on the "Register" link on the right hand side of the website. Only one answer is correct for each question. Once you successfully answer all post-test questions you will be able to view and/or print your certificate. For questions regarding the content of this activity, contact the accredited provider, CME@medscape.net. For technical assistance, contact CME@webmd. net. American Medical Association's Physician's Recognition Award (AMA PRA) credits are accepted in the US as evidence of participation in $\mathrm{CME}$ activities. For further information on this award, please refer to http://www.ama-assn.org/ ama/pub/about-ama/awards/ama-physicians-recognition-award.page. The AMA has determined that physicians not licensed in the US who participate in this CME activity are eligible for AMA PRA Category 1 Credits ${ }^{\mathrm{TM}}$. Through agreements that the AMA has made with agencies in some countries, AMA PRA credit may be acceptable as evidence of participation in CME activities. If you are not licensed in the US, please complete the questions online, print the AMA PRA CME credit certificate and present it to your national medical association for review.

\section{Activity evaluation: where $\mathbf{1}$ is strongly disagree and $\mathbf{5}$ is strongly agree.}

$\begin{array}{lllll}1 & 2 & 3 & 4 & 5\end{array}$

The activity supported the learning objectives.

The material was organized clearly for learning to occur.

The content learned from this activity will impact my practice.

The activity was presented objectively and free of commercial bias.

1. You are seeing a 30-year-old man referred to your clinic for partial epilepsy with epileptogenic foci in both temporal lobes. He has experienced failure of several trials with medical therapy. You consider that he may be a candidate for an implanted device to treat his epilepsy. What should you consider regarding the vagus nerve stimulator (VNS)?

A Stimulation intensity does not correlate well with seizure control

B Approximately one-half of patients experience a long-term reduction in seizures of $50 \%$ or more

C VNS is usually preferred for patients with generalized vs focal epilepsy

D The main adverse effects of VNS include fatigue and depression

2. Which of the following statements regarding responsive neurostimulation (RNS) is most accurate?
A The RNS system is ideal for patients with multifocal epilepsy
B Generally, RNS does not require patient input or activity
C The expected battery life of RNS is 10 years
D Long-term responses are superior to short-term results in RNS

3. You also consider whether to initiate deep brain stimulation (DBS). What should you consider regarding this treatment?

A The most effective brain area to target is the centromedian nucleus

B DBS has been approved in Europe but not the United States

C Patients with extratemporal epilepsy are most likely to respond

D The most common serious adverse effect of DBS is intracranial hemorrhage 
4. What can you tell this patient about other emerging treatments of epilepsy?

$\square$ A The transcutaneous trigeminal nerve stimulation system is meant to be worn at least 12 hours per day

B Transcranial magnetic stimulation should be applied at the vertex without specific targets

C Transmeningeal drug delivery allows for periodic drug infusion

$\square$ D Transcranial direct current stimulation has not been demonstrated to reduce seizure frequency 